5. ESTADO Y CULTURA EN EL ORDENAMIENTO DE LA ITALIA REPUBLICANA

SARA VOLTERRA

Universidad de Padua. Italia 
SUMARIO

1.-Las normas sobre la Cultura en la Constitución de 1948. II.-EXAMEN DEL ARTICULO 9 DE LA CONSTITUCIÓN. III.-COMO SE ENTIENDEN LAS COMPETENCIAS PROMOTORAS DEL ESTADO EN EL ÁMBITO DE LA CULTURA. IV.-ADSCRIPCION DE LA CULTURA EN LA ESTRUCTURA ADMINISTRATIVA. V. - LA PROTECCIÓN DEL MEDIO AMBIENTE EN EL ORDENAMIENTO CONSTITUCIONAL. VI.-EL CONCEPTO DE PAISAJE Y BIENES CULTURALES EN LA CONSTITUCIÓN. 


\title{
5. ESTADO Y CULTURA EN EL ORDENAMIENTO DE LA ITALIA REPUBLICANA
}

\author{
POR \\ SARA VOLTERRA \\ Universidad de Padua. Italia
}

\section{LAS NORMAS SOBRE LA CULTURA EN LA CONSTITUCION DE 1948}

En el ordenamiento que se origina en la Constitución de 1948 no abundan las normas constitucionales referidas a la temática que aquí nos interesa, al igual de lo que ocurre en la casi coetánea Grundgesetz de la República Federal de Alemania; la materia se contempla en menor medida que en la Constitución española de 1978.

La primera de estas normas se encuentra recogida en el Título correspondiente a los principios fundamentales y consta de dos apartados:

\section{Artículo 9}

La República promueve el desarrollo de la cultura y la investigación cientifica y técnica.

Protege el paisaje y el patrimonio histórico y artístico de la nación.

En el Título II, relativo a las relaciones ético-sociales, encontramos dos de las disposiciones más largas de nuestra Constitución: los artículos 33 y 34 . El texto de los dos enunciados es el siguiente:

\section{Artículo 33}

El arte y la ciencia son libres, como libre es su enseñanza.

La República enuncia las normas generales sobre la enseñanza e instituye escuelas estatales de todos los órdenes y grados. 
Entidades y privados tienen el derecho de fundar escuelas a institutos de educación sin cargas onerosas para el Estado.

La ley, en la distribución de derechos y obligaciones de las escuelas no estatales que desean la paridad, habrá de asegurar plena libertad a las mismas y a sus alumnos un tratamiento escolar equivalente al de los alumnos de la escuela pública.

Será necesario un examen de Estado para el ingreso en los diversos órdenes y grados de la escuela o para la conclusión de la misma, así como para la habilitación profesional.

Las instituciones culturales de alto nivel, Universidad y Academia, tienen el derecho de dotarse de ordenamientos autónomos dentro de los límites establecidos por las leyes estatales.

\section{Artículo 34}

La escuela está abierta a todos.

La enseñanza inferior, a impartir como mínimo durante ocho años, es obligatoria y gratuita.

Los capaces y meritorios, aunque se encuentren privados de medios, tienen el derecho de alcanzar los grados superiores de estudio.

La República hará efectivo este derecho por medio de las becas de estudio, de los subsidios familiares y otras asignaciones, que deberán ser atribuidas por concurso.

Además de estos tres artículos se encuentran otros, como, por ejemplo, el artículo 30.2, que implican que la República tiene competencias para favorecer la enseñanza latu sensu. De hecho, estos artículos prevén que serán desarrolladas por la República aquellas atribuciones de enseñanza y educación que constituyen la obligación de los padres, aunque éstos no accedan a tales competencias. Sustancialmente esta norma se halla vinculada a valores formativos de otra naturaleza que los didácticos. En este sentido, la República tiene la atribución, como se deduce del artículo 35.2, de procurar la formación de los trabajadores. También se encuentran las competencias culturales atribuidas a las regiones, que habrán de ejercerse dentro de los límites de los principios fundamentales establecidos por las leyes estatales. Tampoco habrán de ignorarse las disposiciones referidas a derechos culturales contenidas en los estatutos regionales ${ }^{1}$.

1 Las competencias regionales, desde la óptica legislativa, son de tres tipos: primaria o exclusiva; concurrente o compartida; integradora-alternativa. Las regiones ordinarias disponen, en el ámbito cultural y escolar, de competencia concurrente, debiendo respetar los principios impuestos por la legislación estatal. Las regiones de estatuto especial, por el contrario, disponen, en diversa medida y respecto de sectores específicos de la enseñanza y de la cultura, de los tres tipos de competencia, la exclusiva o primaria, la concurrente o 
El número relativamente escaso de artículos referentes al tema de la cultura en el conjunto del sistema no puede llamarnos a engaño. Aunque nos limitemos a las disposiciones de los artículos 9, 33 y 34 de la Constitución, se entiende que a través de la vinculación con el artículo 3.2 (principio de igualdad social), las disposiciones antes citadas se destacan entre aquellas más importantes para calificar el actual sistema constitucional italiano como ejemplo de ordenamiento de democracia social $^{2}$.

compartida y la integradora. Desde el principio los estatutos de las regiones especiales han contenido normas relativas a la cultura y al sector escolar.

El artículo 117.1 de la Constitución obliga al Estado a emanar las leyes-marco con los principios generales dentro de lọ que las regiones ejercitarán la potestad legislativa, respetando también determinados límites que no precisamos enumerar aquí. Indicaremos, por otro lado, que en el momento de escribir este artículo, el número de leyes-marco promulgadas es muy escaso.

Según el artículo 17 de la Ley de financiación regional (Ley n. 281 de 16 mayo 1970), los principios fundamentales no expresamente enunciados por leyes estatales, habrán de recabarse de la legislación vigente.

Diversos problemas, no menos complejos, al producirse también aquí considerables retrasos, han sido planteados por la transferencia y delegación de funciones administrativas.

El trasvase de funciones que hubiese debido realizarse por sectores orgánicos materiales, según lo dispuesto en el artículo 17 de la Ley $\mathrm{n}$. 281 de 16 de mayo de 1970, se efectuó por medio de los decretos legislativos de 1972, de acuerdo con una interpretación restrictiva de las materias de competencia regional. Con la Ley $n$. 382 de 22 julio 1975, se ha operado una nueva delegación al Gobierno, a la que se ha dado realización con el DPR n. 616 de 24 julio 1977. Con este último decreto, como mínimo, se ha reinterpretado la materia, individualizando cuatro sectores orgánicos entre los cuales operar transferencias y delegación de funciones. Por lo que afecta a nuestro tema, nos interesan los sectores del ordenamiento y de la organización administrativa referentes a los servicios sociales y a la determinación y utilización del territorio.

Para estos temas cfr.: E. Spagna-Musco, lo stato di cultura nella Constituzione italiana, Napoli, 1961, págs. 107 y ss.; L. PALADIN, Diritto regionale, Padova, 1979, págs. 8 y ss., 65 y ss., 86 y ss., passim, págs. 174 y ss., passim; T. MARTines y A. Ruggeri, Lineamenti di diritti regionale, Milano, 1984, págs. 153 y ss., págs. 233 y ss., passim; A. BARBERA y F. BASSANINI (recopiladores) / nuovi poteri delle regioni e degli enti locali: Comentario al decreto 616 di attuazione dellla 1382, Bologna, 1978, págs. 21 y ss.; E. CAPACCIOLI y F. SATTA (recopiladores), Commento al decreto 616 (DPR 24 luglio 1977 n. 616), Milano, 1980, páginas 794 y ss., págs. 809 y ss., passim.

Para ejemplificar las normas de los Estatutos regionales referentes al campo cultural podemos citar, limitándonos a unos pocos ejemplos, el artículo 7 del Estatuto de la región de Puglia. Este artículo contempla un uservicio de escolarización idóneo para remover los obstáculos que limitan de hecho el derecho al estudion. A su vez, las leyes regionales dan concreción a las tareas y a los objetivos de las regiones en materia de cultura. Podemos citar, limitándonos a algún ejemplo, la ley regional toscana n. 61 de 31 de mayo de 1975, relativa a la Consulta regional toscana sobre los bienes culturales, o la ley regional $n$. 16 de 5 de mayo de 1977 de la región Basilicata, relativa a la organización de los servicios y de la actividad cultural. También podemos citar la ley regional $n$. 2 de 7 de enero de 1974 de la región Emilia-Romagna en la que se contienen "primeros instrumentos para la tutela, conservación y valoración de los centros históricos".

2 El artículo 3.2 establece "Es competencia de la República remover los obstáculos de orden económico y social que, limitando de hecho la libertad y la igualdad de los ciudadanos, impiden el pleno desarrollo de la persona humana y la efectiva participación de todos los trabajadores en la organización política, económica y social del país". 
Lo primero que podría subrayarse es, efectivamente, que los artículos 9,33 y 34 de la Constitución se encuentran en estrecha y recíproca conexión entre ellos y también con otros artículos del texto constitucional. Sólo un estudio sistemático de este tipo podrá realmente revelar el valor que aquéllos tienen para el conjunto significante del sistema constitucional. De modo particular, la mejor exposición de ello se opera a la luz del artículo 3.2 de la Constitución.

\section{EXAMEN DEL ARTICULO 9 DE LA CONSTITUCION}

Para iniciar el examen del artículo 9 habrá de observarse, en primer lugar que, en los debates sobre este artículo no se produjeron divisiones entre «los tres espíritus» de la asamblea constituyente. Es decir, no se intercambiaron diatribas entre los constituyentes de cultura católica, los de cultura marxista o los de cultura laica. La misma discusión generada en la subcomisión de la denominada comisión de los 75 y en la Asamblea no fue muy amplia ${ }^{3}$. Como mucho pudo notarse cómo algunos constituyentes, por razones de diversa orientación ideológica (caso de Marchesi o Codignola), manifestaran una cierta desconfianza respecto a las competencias culturales atribuidas a las regiones que se instituían. No obstante esta posición consiguió ser superada en la discusión general de la Asamblea gracias a la oportuna intervención del diputado Lussu.

Como sucedió con otros artículos, también el artículo 9 debe su forma definitiva a la comisión de redacción 4 .

3 La Constitución italiana de 1948 fue elaborada por la Asamblea constituyente elegida por el pueblo el 12 de junio de 1946. La Constitución fue promulgada el 27 de diciembre de 1947, entrando en vigor el 1 de enero de 1948.

La Asamblea estaba compuesta por 556 diputados pertenecientes a varios grupos políticos. Esta había creado de su seno una Comisión constitucional, también denominada Comisión de los setenta y cinco, por el número de sus componentes, cuyo cometido era elaborar un proyecto de Constitución que habría de ser sometido posteriormente a la Asamblea.

La Comisión de los setenta y cinco se dividía, a su vez, en tres subcomisiones. La subcomisión primera se ocupó de los derechos y deberes de los ciudadanos, la segunda del ordenamiento de la República y la tercera de los derechos y deberes socioeconómicos. EI texto, discutido y votado por la Asamblea, fue todavia sometido a una revisión, desde el punto de vista formal, por el Comité de coordinación o redacción. En la sesión del 22 de diciembre de 1947, la nueva Constitución fue aprobada por 453 votos a favor y 62 en contra.

4 El Comité de Coordinación o de redacción, llamado de los dieciocho, fue seleccionado entre los miembros de la Comisión de los setenta y cinco, teniendo como misión la de coordinar los trabajos de las subcomisiones primera y tercera. De hecho cumplió una función más importante, constribuyendo a la forma definitiva de algunos artículos que, posteriormente sometió a revisión formal, una vez que el texto de cada uno de los artículos fue aprobado por la Asamblea. Contrariamente a to acaecido con las otras comisiones y subcomisiones, no se consignaron actas de las sesiones de este comité. 
Sin embargo, tanto en los años de la constituyente como en los sucesivos, no faltó la ironía y la crítica referidas al texto del artículo 9 . Se criticaba el hecho de apelar fuera del preámbulo a algo que podría fundamentar pretensiones no judiciables, aunque sí programáticas, y en las que se incluían fines demasiado generales orientados al futuro legislador. Todavía en los años 50 abundaba el escepticismo sobre estas declaraciones constitucionales, máxime cuando el Tribunal Constitucional había declarado inaceptable la "direccionalidad» (Indirizzo) de la doctrina y de la magistratura ordinaria, por lo que el legislador no sabría contemplarse vinculado por las normas programáticas. Como mucho, siguiendo las sugerencias ofrecidas por la propia Constituyente, referidas a otras normas del mismo tipo y, así mismo, de acuerdo con el criterio del constitucionalista Vezio Crisafulli específicamente referido a este artículo, se podría estar dispuesto a ver en esta disposición un limite a la normativa ordinaria. Expresado en otros términos, el artículo en cuestión podria ser, como máximo, suceptible de provocar la incostitucionalidad de leyes que persiguiesen fines contrarios a sus disposiciones ${ }^{5}$.

Consiguientemente la propia operatividad del artículo 9 de la Constitución ya fue cuestionada desde un principio. No obstante, como hemos indicado anteriormente, el citado artículo de la Constitución está considerado como un elemento del conjunto de la misma. Esencialmente dicho artículo es, como algunos otros, una especificación del artículo 3.2.

Conviene, por tanto, considerarlo ahora en sus elementos integrantes, comenzando por el primer apartado: «La República promueve el desarrollo de la cultura y la investigación científica y técnica". Este primer apartado parece haber interesado a la doctrina menos que el segundo, aunque se ha afirmado que el primero constituye la parte más importante del artículo considerado en su totalidad ${ }^{6}$. Ya volveremos sobre este punto; por el momento detengámonos en los dos verbos que afectan a la República -también volveremos sobre el significado de esta expresióncompetencias al mismo tiempo promotoras y protectoras: la República "promueve el desarrollo de la cultura... protege el paisaje y el patrimonio histórico y artístico".

Las competencias promotoras que se afectan a la República son extensibles a otras expresiones frecuentes no sólo en el Título reservado

5 Cfr. V. Crisafullu, La Costituzione e le sue disposizioni di principio, Milano, 1952, pág. 36; A. ANZON, "ll regime dei beni culturali nell' ordinamento vigente e nelle propspettive di riforma", en Ricerca sull'ambiente. (Recopilación a cargo del Secretariado General de la Cámara de Diputados, Servicio de Estudios, Legislación y Encuestas parlamentarias), Roma, 1975, vol. I, pág. 94 nota 2; L. BARsotTI, "Cultura e ricerca scientifica", en La Costituzione economica: prospettive di riforma dellordenamento economico (investigación ISLE a cargo de M. D'ANTONIO), Milano, 1984, pág. 699. gina 74.

E. Spagna-Musso, Lo stato di cuitura nella Costituzione italiana, Napoli, 1961, pá- 
a los principios fundamentales, sino también en aquellos relativos a las relaciones ético-sociales y a las relaciones económicas.

Podemos ver, así, cómo de vez en cuando la República «promueve" (art. 4.1.; art. 5; art. 35.2; art. 44.1; art. 45.1), o bien, "proteje" (art. 35.1; art. 32.1; art. 37 in fine; art. 47.1), o también "favorece» (art. 45.1; art. 47.2), «anima» (art. 47.1), o tiene "la competencia de» (art. 3.2) $y$ "hace efectivo" (art. 34 in fine).

¿Qué hay de común en estas expresiones todas ellas referidas a la República que nace de la Constitución de 1948? Ciertamente se dota a la República de competencias promotoras e impulsoras y de las de intervención activa derivables de las directrices de solidaridad contempladas en los artículos 2 y 3.2 de la Constitución ${ }^{7}$.

En cuanto a las disposiciones relativas a la cultura adquieren un particular significado a la luz de la forma vigente de Estado, y ello en la medida en que éste se diferencia de los Estados del XIX que garantizaban determinados derechos y disciplinaban determinadas relaciones, pero siempre en el marco del reconocimiento previo en favor de una esfera individual de libertad en contraposición al Estado. De una esfera en cuyo interior el Estado no podría intervenir, abandonándola a la libre actuación del sujeto singular. $O$ aunque fuese considerada materia de su competencia ( $y$ hasta el Estado del XIX reconocía como suya la competencia de instrucción), no era, sin embargo, de tal naturaleza como para estar provista de garantía constitucional ${ }^{8}$. La instrucción era materia del Derecho administrativo y no implicaba, por tanto, exigencias concretas recogidas

7 El artículo 2 de la Constitución establece: "La República reconoce y garantiza los derechos del hombre, sea como individuo singular, sea en las formaciones sociales en las que se desenvuelve su personalidad, y exige el cumplimiento de los deberes inderogables de solidaridad politica, económica y social».

8 La excepción, que confirma esta regla, la encontramos en la “Déclaration des droits des français et des principes tondamentaux de leur Constitution" aprobada por la Cámara de presentantes el 5 de julio de 1815, y en la que el artículo 11 establecía: "Une instruction primaire, indipensable pour la connaissance des droits et des devoirs en société, est mise gratuitement à la portée de toutes les clases du peuple. Les éléments des sciences, des belles lettres et des beaux arts, sont enseignés dans des hautes écoles".

La Constitución francesa de 14 de agosto de 1830 no volvió a incluir las disposiciones de la declaración de la de 1815, pero en su artículo 69 estableció que la ley habría de regular «la instrucción pública y la libertad de enseñanza".

La Constitución francesa de 4 noviembre de 1848, a su vez, al reconocer en el articulo 9 "la libertad de enseñanza", establecía que esta libertad habría de ejercerse "de acuerdo con las condiciones de capacidad y moralidad determinadas por las leyes y bajo la vigilancia del Estado". Vigilancia, que sin excepciones, se efectuará sobre "todos los institutos de educación y enseñanza", como precisaba el último apartado del citado artículo.

En Italia, la Constitución de la República Romana de 1849 proclamaba, a su vez, en su artíciulo 8 el principio de la libertad de enseñanza, reservando a la ley de determinación de los requisitos de "moralidad y capacidad" para el ejercicio de la misma. Pero, como se sabe, esta Constitución fue proclamada en el mismo momento en que las tropas francesas entraban en Roma para restablecer el dominio temporal del Papa, por lo que aquélla no pudo ser aplicada. 
en ley superior alguna. La prosecución de fines culturales no era materia de consideración constitucional.

En la Constitución actual la prosecución de fines culturales es un deber del Estado, que no sólo asume la protección sino también la promoción de la cultura. Las atribuciones promotoras están justificadas únicamente en cuanto que ha de quedar garantizada la libre formación de la personalidad del ciudadano ${ }^{9}$. Esta finalidad contribuye a calificar a la República también como «Estado de cultura» ${ }^{10}$.

\section{COMO SE ENTIENDEN LAS COMPETENCIAS PROMOTORAS DEL ESTADO EN EL AMBITO DE LA CULTURA}

En lo que respecta a cómo son entendidas las competencias promotoras del Estado, hay que recordar que tanto el artículo 9.1 como los artículos 33 y 34 de la Constitución conllevan una polémica con el pasado. Polémica que deduce de las competencias promotoras el pacto de entender los citados artículos en el contexto del artículo 3.2, que contempla las obligaciones de las que el Estado se hace cargo como orientadas a la promoción de la igualdad entre los ciudadanos. Obligaciones que finalizan, además, a través de esta igualdad, en la consecución de la plena participación de todos en la vida social.

La polémica se resuelve, sobre todo -aunque no exclusivamente-, contra un pasado reciente a los ojos de los constituyentes de 1948. Se trata de la polémica contra la cultura de Estado, contra el denominado «Estado ético" contra la ideología de «todo en el Estado, todo por el Estado» de la época fascista. Es por esto por lo que no se hicieron muchas diferenciaciones entre los constituyentes: el elemento unificante de las tres culturas era el antifascismo.

Como muchos otros artículos de la Constitución, el artículo 9.1 polemiza con una consideración de las competencias estatales en tanto que competencias de orden, para subrayar, por el contrario, la esencialidad de los nuevos valores, tales como la salud, el trabajo, el estudio. Pero también dice «no" a la cultura de Estado y a la orquestación del consenso. El artículo se opone así a la organización de la cultura a través del carnet del partido, a través del juramento preliminar para el desarrollo de la actividad intelectual o profesional. Se opone a la gestión de la comunicación social como tenía lugar en la época fascista por obra del Ministerio de Cultura Popular. Contra la experiencia de los dos decenios fascistas

9 E. Spagna-Musso, ob. cit., pág. 55.

10 idem. loc. cit. 
se pone la fórmula literal del primer apartado del artículo 9, de la misma forma que contra esta experiencia se pone la fórmula del primer apartado del artículo 33 de la Constitución. «El arte y la ciencia son libres, como ljbre es su enseñanza». En efecto, el apartado primero del artículo 9 de la Constitución afirma que «la República promueve el desarrollo de la cultura», de donde se admite que la cultura existe ya. La República asume la responsabilidad de favorecer su desarrollo y su incremento, pero no condiciona la existencia misma de la cultura. La propia declaración contenida en el apartado segundo del artículo 9 es, como ha indicado ENRICO SPAGNA-MuSSo, un reflejo sectorial del apartado primero, así como tiene "valor de especificación, aunque sea reforzadora del concepto de cultura», el inciso «y la investigación científica y técnica» ${ }^{11}$.

Está muy claro lo que Constitución no quiere de los poderes públicos mediante este primer apartado. Menos fácil, sin embargo, es traducir en concreto un sistema de incentivos compatibles con lo dispuesto en el mismo apartado.

Se puede mencionar en el marco de un interés público dirigido al desarrollo de la investigación científica la creación y potenciación de centros públicos como el ISPE o el CNR. Se puede recordar también la potenciación del Consejo Nacional de Investigación que se operó con la Ley de 2 de mayo de 1963, n. $283^{12}$. Podemos recordar-punto sobre el que volveremos - la creación del Ministerio para la Riqueza Cultural y Ámbiental. Podemos recordar, más recientemente, la utilización, afín a los clásicos instrumentos del intervencionismo, de los beneficios fiscales previstos en la Ley n. 512 del 2 de agosto de 1982.

Se ha observado que, si bien el articulo 9.1, frase primera, tenía - según una autorizada doctrina - mayor relevancia respecto a la declaración contenida en la frase segunda, ha sido propiamente esta última la que ha suscitado mayor interés entre los estudiosos.

Según FABIO MERUSI la explicación de esta aparente contradicción estaría centrada en el hecho de que el principio de la primera frase quedaba "casi enteramente explicitado" en los artículos 33 y 34 de la Constitución ${ }^{13}$. La verdad es que este tipo de lectura del artículo 9 viene a incrementar la innegable dificultad que el artículo pone para acoplarse a dis-

11 Ver E. Spagna-Musso, ob. cit., págs. 74-75.

12 Sobre este tema ver M. S. GIANNINI, "L'organizzazione della ricerca scientifica", en Riv. Trimestrale Dir. Pubbl. 1966, págs. 1 y ss.; G. DE CESARE, "La ricerca scientifica pubblica: aspetti problematici ed organizzativi", en Riv. Ital. Scienze giuridiche, XIII, 1969, páginas 1 y ss.; M. NigRo, «Lo stato italiano e la ricerca scientifica: profili organizzativi», en Riv. Trim. Dir. Pubbl. 1972, págs. 740 y ss.; F. MeRLONI, “L'organizzazione pubblica della ricerca scientifica fra processi reali e ipotesi di riforma", en Riv. Trim. Dir. Pubbl. 1981, págs. 808 y ss.; 1. Tuccr, "La ricerca scientifica nella Costituzione Italiana», en Rassegna parlamentare $1984,2-3$, págs. 267 y ss.

13 Ver F. MERUSI, "Comento all'art. 9" en Commentario della Costituzione: Principi fondamentali (recopilación de G. BraNCA), Bologna, 1975, pág. 435. 
posiciones que parecerían tener una relevancia preceptiva más inmediata. Pero el riesgo de una lectura semejante del artículo 9.1, frase primera, consiste en dejar en la sombra la sustancial unidad del artículo. LUCIA BARSOTI objeta que no se haya adoptado hasta el momento suficientemente y «de manera global y orgánica una exigencia de actuación" de esta primera fase, de tal modo que ésta, examinada aisladamente respecto del contexto unitario de la disposición, habría producido «una verdadera alteración en los mismos fundamentos de la cuestión cultural, con el efecto de reducir la idea de cultura, en la legislación y en la práctica administrativa, a una mera acumulación de patrimonio cultural» ${ }^{14}$.

\section{ADSCRIPCION DE LA CULTURA EN LA ESTRUCTURA ADMINISTRATIVA}

Antes de proceder al examen de la segunda frase, conviene observar que, mientras que durante casi un siglo la cuestión cultural se ha visto afectada a la gestión del Ministerio de Instrucción Pública, ha sido recientemente creado (decreto legislativo n. 657 de 14 diciembre 1974 y Ley $n .^{\circ} 5$ de 29 enero 1975) el Ministerio de la Riqueza Cultural y Ambiental.

Sobre la noción de riqueza cultural ya volveremos más adelante. Urge aquí subrayar que el procedimiento se inscribia en la lógica burocrática tradicional, esto es, en la lógica de la fragmentación departamental de la Administración ${ }^{15}$, mientras que la más correcta interpretación del artículo 9 de la Constitución impone una estructuración unitaria. Por otro lado, todavía no se había concluido la individualización de los diversos niveles organizativos de intervención pública (Estado, Regiones, Entes locales).

En el momento de la creación del nuevo Ministerio se reenvió a una ley marco futura la individualización de la «distribución... riqueza cultural ambiental» ${ }^{16}$, delegando en las regiones únicamente la gestión de la riqueza ambiental comprendida en la limitada acepción de «bellezas naturales". Quiere ello decir que se siguió más bien el esquema definitorio propio de la época preconstituyente, antes que la del artículo 9 de la Constitución.

Recordemos, además, que las regiones de estatuto ordinario se encontraban ya situadas en condición de promotoras del desarrollo de la cul-

14 Ver L. BARSOTTI, "Cultura e ricerca scientifica", en La Costituzione economica (recopilación de M. D'ANTONIo para el ISLE) Milano, 1985, pág. 699.

15 Cfr. Sobre este punto, por ejemplo, N. GreCo, Stato di cultura e gestione dei beni culturali, Bologna, 1981, pág. 109.

io N. Greco, ob. cit., pág. 26. 
tura. Por tanto, numerosos estatutos de estas regiones (art. 5 Est. Basilicata; art. 56.2 Est. Calabria; art. 3 Est. Emilia-Romagna; art. 4 Est. Molise; art. 5 Est. Marche, etc.) regulaban - más allá de la competencia legislativa concurrente, expresamente establecida para aquélla en el artículo 117 de la Constitución ${ }^{17}$ - la compartición del patrimonio histórico, artístico y cultural.

A su vez, los estatutos de las regiones especiales han atribuido a estas regiones potestad legislativa integral $y$, en algunos casos, hasta exclusiva sobre la "conservación de las antigüedades y de las obras de arte» (art. 5 Est. Sardegna; art. 14 Est. Sicilia; art. 3 Est. del Valle de Aosta, etc. ${ }^{18}$.

\section{LA PROTECCION DEL MEDIO AMBIENTE EN EL ORDENAMIENTO CONSTITUCIONAL}

El apartado segundo del artículo 9 enuncia: «Protege el paisaje y el patrimonio histórico y artístico de la nación». El sujeto ausente es el mismo que el del apartado primero: la República.

Lo primero que podemos observar es que la Constitución italiana no menciona «el medio ambiente» ni en el artículo 9 ni en ningún otro. En ello es similar a la casi coetánea Ley Fundamental de la República Federal Alemana, pero distinta de la Constitución española de 1978 que, como es sabido, contiene una referencia a la cuestión.

Sin embargo, no han faltado en Italia propuestas de un reconocimiento explícito del medio ambiente. Por ejemplo, la Academia sugirió, en 1983, una posible -adición al artículo 9, concebida en los siguientes términos: "La República reconoce a todos los ciudadanos el derecho al medio ambiente y promueve las condiciones que hacen efectivo este derecho, sea en el campo de la información, de la participación o de la acción».

17 El artículo 117 de la Constitución establece que «Las regiones, dentro de los límites de los principos establecidos por las leyes estatales, establecen normas legislativas en las materias que se enumeran a continuación, simpre que aquéllas no se contrapongan al interés nacional o a los intereses de las otras regiones:

- regulación de las funciones y de los entes administrativos dependientes de las regiones;

- circunscripciones municipales;

- policia local urbana y rural;

- ferias y mercados;

- beneficiencia pública y asistencia sanitaria y ambulatoria;

- aprendizaje artesanal y profesional y escolarización;

- museos y bibliotecas de los entes locales;

- urbanística.

18 Ver V. ITALIA, ob. cit., pág. 97, nota 8. 
«El derecho al medio ambiente se ejerce individual o colectivamente en el marco de las leyes que lo regulan. Todo ciudadano tiene el . deber de respetar y conservar los recursos culturales y naturales del país en cumplimiento del principio de solidaridad social y también en consideración al derecho al medio ambiente de las generaciones futuras".

Aquello que no se ha previsto en la Constitución de la República, sí se encuentra recogido, en cambio, en numerosos estatutos regionales en los que se contienen enunciados sobre el medio ambiente. Las razones del silencio respecto a la protección del medio ambiente en la Constitución italiana pueden ser de diversa índole.

Recordemos que también faltaba la referencia a esta cuestión en la formulación del artículo hecha por el ponente Sr. Marchesi en la 1. ${ }^{\mathrm{a}}$ subcomisión de la Comisión de los 75 que redactó la Constitución. De hecho se sugería poner bajo vigilancia estatal "los monumentos artísticos, históricos y naturales». PREDIERI ha observado que el artículo parece copiado de una norma de la Constitución de Weimar, si exceptuamos la referencia del paisaje ${ }^{19}$. Fue la comisión redactora la que substituyó la expresión "monumentos naturales", ya criticada en la comisión, por la de "protección del paisaje» ${ }^{20}$.

Pudo suceder que, independientemente de la intención polémica en confrontación con el régimen anterior, patente en la misma adopción del artículo 9 de la Constitución, no obstante, el constituyente hubiese sentido la influencia de la legislación de 1939 y, en particular, la de la Ley n. 1089 de 1-VII-1939 sobre "protección de las bellezas naturales y panorámicas". Legislación que recogía una noción estática y estetizante del paisaje.

Pußg ser también, que nuestro constituyente no advirtiera el surgimiento de nuevas exigencias que, por otro lado, tampoco se habían afirmado realmente en otros países, toda vez que las mismas no se evidenciaron más que hacia los años 60 . También podría ser que se considerase al término "medio ambiente» excesivamente amplio como para ofrecer la posibilidad de una noción jurídica unitaria. Puede ser -como se observó- que la noción de paisaje tenga puntos de coincidencia estructural «con alguna noción de medio ambiente» ${ }^{21}$.

Ello no quiere decir que no puedan encontrarse aún hoy en la Constitución italiana elementos de conexión entre la problemática de los derechos inviolables y de la dignidad de la persona (enunciados en los

19 Ver A. Predierl, «Patrimonio storico-artistico e tutela del paesaggio", en La Costituzione economica: prospettive di riforma dell ordinamento economico (recopilación de $\mathrm{M}$ : D'ANTONIO para ISLE) Milano, 1985, pág. 713.

20 Ob. y loc. cit.

2) Ver A. Predieri, ob. cit., pág. 714. 
arts. 2 y 3.1 de la Constitución) ${ }^{22}$, y la exigencia de evitar daños al medio ambiente susceptibles de inicidir sobre aquellos valores. La instrumentación jurídica a utilizar no se encontrará en las normas relativas al "Estado de cultura», sino en aquellas que contemplan el derecho a la salud ${ }^{23}$.

Habíamos dicho que el artículo 9 habría de considerarse unitariamente. Ello lleva a intentar justificar lógicamente el vínculo entre la promoción de la cultura del primer apartado y la especificación «paisaje y patrimonio histórico y artístico" del segundo. Para hacerlo debemos vincularlo al diseño solidario que establece la Constitución, recogido en el Título dedicado a los principios fundamentales en sus artículos 2 y 3.2. La cultura es un instrumento para alcanzar el objetivo de "remover los obstáculos de orden económico y social que, al limitar de hecho la libertad y la igualdad de los ciudadanos, impiden el pleno desarrollo de la persona humana y la participación de todos los trabajadores en la organización política, económica y social del país" (art. 3.2). Recordemos que la República «reclama el cumplimiento de los deberes inderogables de solidaridad política, económica y socialı (art. 2). Por tanto, no sólo la protección y la defensa de la riqueza cultural, sino también su valoración y la oferta de su disfrute.

La cultura está directamente tutelada por la Constitución y su promoción se encuentra elevada al rango de principio fundamental, debido a que los principios de solidaridad y de personalidad son elementos clave de la forma de Estado creada por la Constitución.

En este punto, antes de afrontar la noción de paisaje utilizada en la Constitución, y antes de examinar el vínculo entre la protección del paisaje y la del patrimonio histórico y artístico, así como también su vínculo con el concepto de cultura, conviene indagar acerca de la expresión «República» utilizada en el art. 9.

En el texto constitucional son frecuentes las referencias a la República, empezando por el artículo 1, que define a Italia como una «República basada en el trabajo". Limitándonos al uso del término en el Título relativo a los principios fundamentales, observamos que, usualmente, el término se encuentra entendido en un sentido lato, en el que se comprenden todas las articulaciones del Estado ordenamiento y no sólo el Estado persona. Consiguientemente, junto al Estado central, se incluyen

22 Sobre el artículo 2 de la Constitución ver la nota 7. El artículo 3.1 establece que "Todos los ciudadanos poseen igual dignidad social y son iguales ante la ley, sin limitación de sexo, raza. lengua, religión, opinión política o de condición personal o social».

23 El artículo 32 de la Constitución establece que «La República tutela la salud como derecho fundamental del individuo e interés de la colectividad, y garantiza atención médica gratuita a los indigentes. Nadie puede ser obligado a un determinado tratamiento sanitario si no es en virtud de lo dispuesto en las leyes. Las leyes no podrán violar, en ningún caso, los límites impuestos por el respeto a la persona humana". 
también otros entes autónomos, tales como las regiones, los municipios y las provincias. El artículo 5 de la Constitución que dice «La República; una $\mathrm{e}$ indivisible, reconoce y promueve las autonomias locales", implica que el asiento de nuestro Estado no es el mismo que el del ordenamiento precedente. El artículo 114 afirma, efectivamente, que Italia es un Estado regional. "La República se reparte en regiones, provincias y municipios". Este es uno de los pocos casos en los que por necesidad lógica «República" tiene un significado no extensivo. Pero en la mayor parte de los casos -y, sobre todo, en el ámbito de los principios fundamentales-, alli donde se establecen los valores impulsores y personalistas que se encuentran en la base del ordenamiento, las referencias a la República no se limitan al Estado-persona. Recordemos al respecto los ya citados artículos 2 y 3.2 de la Constitución. ¿Podría sostenerse que el objetivo de remover los obstáculos que se opongan a la efectiva igualdad de los ciudadanos constituye una tarea únicamente encomendada a los órganos del Estado-persona? La respuesta ha de ser negativa. Pensemos en el artículo 6 que atribuye a la República la competencia de tutelar las minorías lingüísticas. Hemos de admitir que ha existido la tendencia - mantenida también en el pasado por el. Tribunal Constitucional- inclinada a sostener que tales competencias fuesen sólo propias del Estado-persona. $Y$, de hecho, acaeció que las regiones de estatuto ordinario tuvieron dificultades para ejercer la tutela de las culturas minoritarias debido a la oposición de los comisarios del Gobierno, que negaban su aprobación a las leyes regionales en dichas materias ${ }^{24}$. En la actualidad, el Tribunal Constitucional, si bien de un modo prudente, parece revisar la instauración de su fase anti-regionalista, reconociendo que la protección de las minorias no es «una materia» a la medida del artículo 117 de la Constitución, sino una directriz para toda la articulación del ordenamiento ${ }^{25}$. República queda entendida aquí entonces de un modo extensivo.

24 En el sistema regional italiano todas las leyes aprobadas por el Consejo regional son comunicadas al Comisario del Gobierno dentro de los cinco días siguientes a su aprobación, disponiendo aquél de treinta días para dar su visto bueno. El citado Comisario dará a conocer al Gobierno central el texto de las leyes aprobadas por las regiones. Estas leyes podrán ser devueltas al Consejo regional para una nueva deliberación. El Consejo regional podrá renunciar al texto legislativo o ratificarlo por mayoría absoluta. En este último caso, el Gobierno, en los quince dias siguientes, podrá impugnar el acto ante el Tribunal Constitucional por razones de legitimidad, o ante las Cámaras por vicio de contenido. El "visto bueno" del Comisario del Gobierno es el medio por el que el Gobierno central consiente a la subsiguiente promulgación de las leyes regionales.

Otro tipo de problemas $\rightarrow$ que no podemos abordar aqui- son los que se originan en el caso de modificación del texto originariamente aprobado.

Respecto de todas estas cuestiones remitimos a la doctrina que citamos a continuación. Ver: L. Paladin, Diritto regionale, Padova, 1979, págs. 384 y ss.; T. MARTInes y A. RuGGERI, Lineamenti di diritto regionale, Milano, 1984, págs. 318 y ss.

${ }_{25}$ Permítasenos remitir a: S. VOLTERRA, «Appunti sulla tutela giuridica delle minoranze linguistiche in Italia ed in altri paesi", en Atti del Convegno sobre "Lingua e Cultura", Cagliari 10-12 de mayo, 1984 (en impresión) y a la bibliografía aquí citada. 
Hagamos notar, no obstante, al examinar el rol de la República en la actuación del "Estado de cultura" y de los artículos a él referidos, que el considerable retraso con que se constituyeron tanto las regiones de estatuto especial como las de estatuto ordinario, no dejó de incidir negativamente en la relación Estado-cultura.

Recordemos que, aún no hace muchos años, una parte de la doctrina sostenía que las regiones de estatuto ordinario habían obtenido demasiado poco espacio en lo tocante al campo de la cultura, en cuanto que la potestad legislativa concurrente se encontraría circunscrita a las materias recogidas en el artículo 117, tales como, por ejemplo, museos y bibliotecas de los entes locales y las competencias en el sector urbanístico ${ }^{26}$. El discurso de las regiones de estatuto especial era distinto, en la medida en que los estatutos de estas regiones atribuian a éstos, en formulaciones no homogéneas, bien una competencia legislativa primaria, bien competencias integradoras en el ámbito cultural ${ }^{27}$. Se observó, sin embargo, que las regiones de estatuto ordinario habían ampliado en sus estatutos el espacio que, en el campo cultural, les atribuia el artículo 117 de la Constitución. Se percibía la persistente ausencia de una ley-marco del Estado. Afirmar que los estatutos de las regiones de autonomía ordinaria que se proponían el objetivo de la valoración y apreciación del patrimonio histórico, artístico y natural (art. Est. Piamonte; art. 3 Est. Lombardia; art. 4 Est. Toscana, etc.) habian interpretado extensivamente las normas constitucionales, era algo que sólo podía ser compartido si se admitía que el artículo 9 únicamente consideraba al Estado-persona como sujeto competente de la actividad promotora de la cultura.

Consiguientemente, podía sostenerse muy bien, que aquellos estatutos regionales, al garantizar los derechos culturales, daban vía de realización a lo dispuesto en el artículo 9 de la Constitución.

Señalemos, no obstante, que aún después de la instauración de las regiones de estatuto ordinario, no faltaron por parte del Tribunal Constitucional, especialmente en los primeros años de la década de los setenta, lecturas restrictivas de las competencias de las regiones de estatuto ordinario, sobre todo en relación a aquellas que, como veremos, son competencias exigidas a la República por el segundo apartado del artículo 9. A título de ejemplo citaremos la exclusión del ámbito competencial regional de la protección de las bellezas naturales; la exclusión de la protección del paisaje, a no ser el paisaje incluido en la relación de materias contenidas en el artículo 117 de la Constitución. Citaremos también una

26 Ver el artículo 117 de la Constitución citado en la nota 17. Sobre estas posiciones ver A. ANZON, cll regime dei beni culturali nell' ordinamento vigente e nelle prospettive di riforma", contenido en Ricerca sull'ambiente (recopilación a cargo del Secretariado general de la Cámara de Diputados, Servicio de Estudios, Legislación y Encuestas parlamentarias) Roma, 1975, págs. 163 y ss.; V. ITALiA, ob. cit., págs. 96 y ss.

${ }_{27}$ Ver la doctrina citada en la nota precedente. 
lectura de las materias urbanísticas (atribuidas a $_{\text {las }}$ regiones) aunque referidas a las edificaciónes cen los centros trabitadôs" (STC n. 141 de 24 julio 1972). Citaremos además da exclusión de las átribuciones relativas a la agricultura, exigible a la competencia régional en función de sus atribuciones en la submateria de parqués (STC n. 142 de 24 julio 1972). Con todo, en este último casó se hạ prodưcido una evolución sucesiva de la jurisprudencia más favorable a las regiọnes y a los entes locales (STC n. 175 de 14 julio 1976).

Por otro lado, hay que tener ến "cuénta la redistribución de materias intervenidas después de la selección operada por el DPR n. 616 de 24 julio $1977^{28}$. Se aprobaron nuevas concesiones relativas a la defensa de la riqueza ambiental, siendo delegadas a las regiones funciones importantes. Tampoco hay que olvidar que el Tribunal Constitucional en la sentencia n. 239 de 1982 ha afirmado que la tutela del paisaje -refiriéndose aquí al apartado segundo del artículo 9 de la Constitución- «es competencia de la República y, consiguientemente, en primer lugar del Estado", con lo que evidentemente se quiere aludir no sólo al Estadopersona, sino también a las regiones y a los entes locales.

\section{EL CONCEPTO DE PAISAJE Y BIENES CULTURALES EN LA CONSTITUCION}

Conviene ahora plantearse la cuestión de cuál sea el concepto de paisaje que enuncia la Constitución. Igualmente conviene preguntarse cuáles sean las razones de su tutela conjuntamente con la de la riqueza histórico-artística.

Según la definición proporcionada por AlBerto PREDIERI, el paisaje es «expresión de una dinámica de fuerzas naturales, pero igualmente y, sobre todo, de fuerzas humanas que actúan permanentemente» ${ }^{29}$. De aquí que, aparte de un hecho físico objetivo, «el paisaje» es «un proceso creador continuo" ${ }^{30}$. En otros términos, constituye "la forma visible del territorio o del entorno, creado por la comunidad humana que se inscribe

28 Cfr. A. BARBERA y F. BASSANINI "ll decreto 616 fra riforma delle autonomie locali e riforma dell'administrazione centrale", en I nuovi poteri delle regioni e degli enti locali: commentario al decreto 616 di attuazione delle 1382 (recopilación de A. BARBERA y F. BASSANINI) Bologna, 1978, págs. 21 y ss.

29 Ver A. PREDIERI, «Significato della norma costituzionale sulla tutela dei pasaggio», en Studi per il XX anniversario dell'Assemblea Costituente: le libertà civili e politiche, Firenze, 1969, vol. 2, págs. 21 y ss.

3o Ver ob. ult. cit. pág. 387. Ver también A. Predieri, voz «Paesaggio", en Enciclopedia del diritto, vol. XXXI, Milano, 1981, págs. 506 y ss. 
en él en una interacción continua entre la naturaleza y el hombre» ${ }^{31}$. Esta concepción del paisaje resulta fundamental para justificar aquella consideración conjunta del patrimọnio histórico-artístico y del paisaje que se enuncia en el artícúlo 9 de la Constitución.

En la actualidad se difunde cada vez más -extramuros de la Constitución - la noción de medio ambiente. Pór ejemplo en el artículo 82 del DPR n. ${ }^{\circ} 616$ de 1977 con objeto del reparto de competencias entre el Estado y las regiones y dè la delegación a las regiones. Sobre esta última noción la doctrinä se encuentra dividida entre aquellos que consideran el medio ambiente como un concepto relacional, no suceptible de prestarse a definiciones jurídicas unitarias ${ }^{32}$, y aquellos otros que, por el contrario, mantienen la posibilidad de una definición jurídica unitaria ${ }^{33}$. Sin entrar en la discusión relativa a la cuestión de las definiciones, podemos adoptar, como coherente con el modelo de Constitución, el concepto de cultura elaborado por AMADEO POSTIGLIONE, para el que " "cultura" es no sólo el producto del hombre, lo que constituye "testimonio de la civilidad" en el territorio, los bienes culturales (muebles e inmuebles), sino la relación con la totalidad de los recursos" ${ }^{34}$.

Podemos observar que a pesar del considerable avance conseguido con el DPR $n .^{\circ} 616$ y los numerosos estatutos regionales en los que se incluyen importantes enunciados referentes al artículo 9 de la Constitución, todavia no se ha realizado adecuadamente el compromiso de las regiones y de los entes locales en la gestión del territorio y de los bienes culturales. (La ley-marco, que ya debería estar promulgada, aunque se propuso el 31 de diciembre de 1979, todavía no ha sido aprobada). Podemos decir que nos encontramos aún en los inicios de una concepción del territorio como lugar designado para el ejercicio de una multiplicidad de funciones, en parte atribuidas a las regiones y a los entes locales, con fines de promoción y salvaguarda de los bienes histórico-artísticos y ambientales.

Es evidente que si los poderes públicos han de regular las modificaciones del territorio, habrán de mantener y hasta incrementar el valor de los bienes culturales que constituyen el patrimonio histórico y artístico del mismo. En otros términos, no debe haber disociación alguna entre desarrollo del medio ambiente, entendido en su totalidad, y defensa de los bienes culturales. Esta última expresión no se encuentra utilizada en la

31 A. Prediefl, «Patrimonio storico-artistico e tutela del paesaggio", en La Costutizione economica: prospettive di riforma dell'ordinamento economico (recopilación de A. D'antonio) cit. pág. 714.

32 Ver M. S. GianniNI, “Ambiente: saggio sui diversi aspetti giuridici», en Riv. Trimestrale Dir. Pubblico, 1973, págs. 23 y ss.; G. Morbidelli, "La propietà. II governo del territorio", en Manuale di Diritto Pubblico, Bologna, 1984, págs. 888.

33 Ver A. POSTIGLIONE, «Ambiente: suo significato giuridico unitario», en Riv. Trimestrale Diritto Pubblico, págs. 32 y ss. págs. 57 y ss.

34 Ver A. Postiglione, ob. cit., pág. 55. 
Constitución, aunque ha sido adoptada tanto por la ley que instituye el Ministerio de la Riqueza Cultural y Ambiental, como por el DPR n. ${ }^{\circ} 616$.

Se observa que en el DPR $n .^{\circ} 616$ se toman precauciones inter alia respecto al orden del territorio y a la protección de la naturaleza (art. 83), a la protección de los bienes culturales (art. 48), al establecimiento de escuelas estatales (art. 46) y a la actividad de promoción educativa y cultura (art. 49). También se presta atención a las disciplinas literarias, musicales y cinematográficas (art. 40).

Por lo que atenta a los bienes culturales se establece, en todo aquello que afecta a las funciones administrativas de las regiones y de los entes locales respecto de la valoración del patrimonio histórico, bibliotecario, artístico, arqueológico, monumental, paleoetnológico y antropológico, el reenvío a aquella ley-marco que aún no ha sido emanada.

La noción de "bienes culturales» ha sido elaborada por la comisión de estudio para la protección y valoración del patrimonio históri$\mathrm{co}$, arqueológico y artístico y paisajístico que comienza sus actuaciones en 1964 y concluye sus trabajos en 1966 (Comisión Franceschini) ${ }^{35}$. Serán bienes culturales aquellos "bienes que constituyen testimonio material con valor de civilidad". En varias de sus obras, M. S. GIANNINI ha realizado un amplio análisis crítico del concepto ${ }^{36}$.

$\mathrm{Si}$, de acuerdo con GIANNINI, entendemos la noción de «bienes culturales" como un umbral jurídico, dado que precisa del reenvío a otras disciplinas para dotarla de contenido ${ }^{37}$, no se le puede negar, sin embargo, una validez científica. Es innegable que los bienes ambientales pueden ser clasificados como bienes culturales.

De hecho pueden considerarse puramente marginales los bienes ambientales estrictamente naturalista, esto es, aquellos no sujetos históricamente a la intervención modificadora del hombre, si no es el hecho de hacerlos accesibles ${ }^{38}$.

Se prodrían habilitar formas diversas de protección a través de la ley 1089/1939 sobre cosas de interés artístico e histórico, o a través de la ley $1497 / 1939$ sobre las bellezas naturales, o bien a través de los medios de tutela previstos en el artículo 48 DPR 616; se podrían usar los instru-

35 Ver F. FranceschinI, «L'impegno della Costituzione italiana per la salvaguardia dei beni culturali", en Studi per il XX Anniversario dell'Assemblea Costituente: Le libertà civili e politiche, Firenze, 1969, vol. 2, págs. 235 y ss.; A. Anzon, ob. cit., págs. 97 y ss.; N. Greco, Stato di cultura e gestione dei beni culturali, págs. 189 y ss.

$1976^{1}$, págs. 3 y ss.

37 Ob. ult. cit. pág. 8.

38 Ob. ult. cit. págs. 11 y ss., pág. 13. 
mentos urbanísticos, pero el vínculo entre las categorías de bienes ambientales y de bienes culturales es innegable, hasta tal punto que ya no se rechaza la necesidad de "una consideración unitaria» 39 .

\section{ANALISIS DE LOS ARTICULOS 33 Y 34 DE LA CONSTITUCION}

Conviene ahora abordar expresamente los dos artículos 33 y 34 , que inciden sobre las cuestiones de la escuela, la cultura y la investigación científica.

El artículo 33, que ya habíamos citado al comienzo de nuestra exposición, es uno de los más largos de nuestra Constitución. Dicho artículo plantea una serie de interrogantes al intérprete. Aquí sólo nos detendremos en algunos de estos problemas.

Las disposiciones del artículo 33 (y del artículo 34) son consideradas por una parte de la doctrina como una explicitación del artículo 9 de la Constitución ${ }^{40}$, mientras otra parte niega esta interpretación, alegando la diferente génesis de estos artículos en la Asamblea constituyente ${ }^{41}$. El problema es determinar si en la expresión, la República, que enuncia las normas generales sobre instrucción (art. 33.2), se incluye, como en el artículo 9 de la Constitución, a las regiones y a los entes locales, o si sólo se limita a los órganos del Estado central. Si centramos este problema en el apartado primero del artículo 33, alli donde se enfatiza que "El arte y la ciencia son libres, como libre es su enseñanza", no puede negarse entonces la complementaridad con el apartado primero del artículo 9 de la Constitución. En ambos apartados se excluye el principio de la oficialidad de la ciencia ${ }^{42}$.

No obstante, en sentido estricto, se podría decir que lo dispuesto en el apartado primero del artículo 33 de la Constitución, es un pleonasmo de lo que se garantiza en el artículo 24 de la Constitución relativo a la libre manifestación del pensamiento ${ }^{43}$ y que, sin duda, ha querido

39 A. Postiglione, ob. cit., pág. 38; G. Morbidelli, ob. cit., págs. 889 y ss.

40 Ver, por ejemplo, F. MERusi, ob. cit., págs. 435 y ss.

41 Ver, por ejemplo, S. CASSESE y A. MURA, Commento allart. 33 y 34 Costituzione: Rapporti etico-socialiti (recopilación de A. BRANCA) Bologna, 1976, págs. 259 y ss.; A. MURA, "Scuola, cultura e ricersa scientifica", en Manuale di Diritto Pubblico (recopilación de G. Amato y A. Barbera), Bologna, 1984, págs. 991 y ss.

$4_{2}$ Cfr. G. DE CESARE, ob. cit., pág. 12.

43 El artículo 21.1 de la Constitución establece que sTodos tienen el derecho de manifestar libremente el propio pensamiento de palabra, por escrito o por cualquier otro medio de difusión». En el apartado seis de este articulo se establece que «Quedan prohibidas las publicaciones, espectáculos y cualquier otra manifestación contraria a las buenas costumbres. La Ley establecerá los adecuados procedimientos para prevenir y reprimir las violaciones". 
reafirmarse en los artículos dedicados a la escuela. Va de suyo que la libertad de enseñanza "sobrepasa los confines de la escuela" ${ }^{44}$.

Antes de abordar la cuestión del significado del término «República» en los artículos sobre la escuela, observamos que se contemplan dos tipos de libertad en el artículo 33. La libertad de la escuela y la libertad en la escuela. La Constitución, como se deduce del apartado tercero del artículo 33, respeta al máximo la libertad y la autonomía de los privados (en conexión con el art. 30, que reconoce el derecho de los progenitores a educar e instruir a sus hijos) en la elección de las directrices educativas (principio de pluralismo escolar) (art. 33.3 de la Constitución). Ello comporta, evidentemente, el derecho de entidades y de privados para fundar escuelas e institutos de enseñanza. La escuela privada puede ser también de naturaleza confesional. Las entidades y los privados siempre podrán fundar escuelas, dado que éstas no conllevan "cargas onerosas para el Estado" (art. 33.3). El Estado está obligado a fundar escuelas de todos los órdenes y grados (art. 33.2) ${ }^{45}$.

Contrariamente a cuanto expusimos en torno al artículo 9 de la Constitución, la totalidad del artículo 33 -independientemente de los acuerdos preventivos, también aquí debidos al rechazo por los partidos antifascistas de la escuela del pasado- es el fruto de una contraposición y de un compromiso. Los católicos se oponían al "protagonismo del Estado en el sector educativo" ${ }^{46}$, debido a su convicción de que la función educativa compete principalmente a la familia. Los sectores laicos y los partidos de izquierda pretendían reservar al Estado la capacidad determinante en el desarrollo de la instrucción. De aquí que la función y el servicio públicos de la enseñanza sean objeto de garantía constitucional. Garantía que también se expresa confiriendo al Estado la facultad de vigilar para que otros valores constitucionales, tales como la seguridad, la libertad y la dignidad de la persona, se encuentren tutelados. Compete al Estado asegurarse de la equivalencia y paridad de la instrucción impartida, en tanto que sólo el Estado posee la facultad de expedir títulos con valor legal. La verificación del grado de cultura y de capacitación profesional habrá de efectuarse por medio de un examen de Estado, y ello con el fin de garantizar los intereses permanentes de la comunidad.

44 Ver V. Grisafulu, "La scuola nella Costituzione», en Riv. Trimestrale Diritto Pubblico, 1956, pág. 69.

45 Se trata de una obligación del Estado-persona. Recordemos que, de acuerdo con la ley n. 820 de 24 de septiembre de 1971 (art. 11), habrán de instituirse escuelas elementales en todas las localidades en cuyo radio de acción de $2 \mathrm{Km}$. se encuentren al menos diez alumnos. Escuelas medias inferiores - según el articulo 10 de la ley n. 1859 de 31 de diciembre de 1969- habrán de fundarse en todos los Municipios con una población superior a treinta mil habitantes. La ley n. 1859 instituyó en todo el territorio de la República la denominada "escuela media única" con una duración de tres años. Por ello, a este nivel, la enseñanza es la misma en las distintas escuelas.

46 Sobre este punto ver G. RICUPERATI; vOz "Scuola", en "Storia d'Italia", en /I Mondo Contemporaneo, Firenze, 1978, vol. 3, pág. 1201. 
Pero volvamos a la primera exigencia: ¿qué quiere decir "La República enuncia las normas generales sobre la instrucción e instituye escuelas estatales de todos los órdenes y grados?" (art. 33.2). Gran parte de la doctrina estima que, en este caso, la expresión "La República", diferentemente de lo que, por lo general, ocurre en el texto constitucional, "alude al Estado-persona» 47. G. STADERINE, en cambio, se opone a esta construcción, al considerar que lo dispuesto en el segundo apartado no se refiere a la instrucción pública - ya que al Estado no le puede impedir la regulación detallada del servicio de instrucción pública-, sino, más bien, a la instrucción privada ${ }^{48}$.

La elección entre estas dos posiciones permite obtener una respuesta distinta a la relación entre la instrucción y las regiones. Recordemos que el artículo 117 de la Constitución reconoce a las regiones de estatuto ordinario la competencia legislativa en materia de escolaridad y de enseñanza profesional y artesana. Las regiones no podrán emanar normas legislativas en las materias de su competencia más que en ámbito determinado por los principios fundamentales que, dada la ausencia de leyes-marco estatales, vendrán derivados del artículo 17 de la Ley 281 (16 de julio de 1970). El artículo en cuestión delega al Gobierno el poder para emanar, durante un bienio, decretos legislativos con las oportunas normas legales para regular el traspaso a las regiones de las funciones atribuidas. No podemos abordar aqui los grandes problemas que surgen, entre otras cosas, porque el artículo 17.1 se refiere también a las funciones administrativas.

Los decretos de delegación han sido emanados tanto en 1972 , como en 1975 y en 1977, transfiriéndose, así, a las regiones la materia de "escolaridad" con el DPR n. 3 de 14 de enero de 1972, y la de ensenanza profesional y artesana con el DPR n. 10 de 15 de enero de 1972. Fueron introducidas importantes innovaciones por medio de la Ley $n$. 382 de 22 de julio de 1975 y, después, por medio del DPR n. 616 de 1977.

La competencia legislativa regional se configura sobre el trasvase de funciones administrativas. Las transferencias se han efectuado en el ámbito de cuatro sectores orgánicos: ordenamiento administrativo, territorio, servicios sociales y desarrollo económico. El territorio y los servicios sociales afectan a la cultura. El territorio por todo cuanto ya hemos mencionado sobre el paisaje, mientras que, en el ámbito de los servicios sociales, entra el sector de la instrucción y de la escolaridad (art. 42), pero también entra la materia de los bienes culturales (art. 47: museos y bi-

47 Ver V. Crisafulli, La scuola, cit., pág. 63. Para una opinión análoga, ver G. CaloGERO, "La scuola, le scienze e le arti», en Commentario sistematico della Costituzione (recopilación de A. Calamandrei y A. LeVI), Firenze, 1950, vol. 1, pág. 325; E. Spagna-Musso, Lo Stato di cultura, cit. pág. 118; A. MURA, Scuola, cultura e ricersa scientifica, cit. pág. 992.

48 F. StaderinI, Osservazioni in tema di norme generali sull'istruzione' en Riv. giurudica della scuola, 1968, págs. 607 y ss. 
bliotecas de los entes locales: art. 48: bienes culturales; art. 49: actividades de promoción educativa y cultural), con tanto más razón que en el cap. VI, la escolaridad, del artículo 42 . Esta materia se halla vinculada, como veremos, a lo dispuesto en el artículo 34 de la Constitución.

En lo que afecta a las regiones, no se puede hablar de extensión de las atribuciones regionales en materia de cultura, o en lo dispuesto en el apartado primero del artículo 33 de la Constitución, dado que se trata de funciones que el ordenamiento exige de todos los sujetos.

Si se mantiene que la expresión «la República» en el apartado segundo y en los otros apartados del artículo 33, así como en los del artículo 34 de la Constitución, alude únicamente al Estado-persona, podemos entonces afirmar que en la actualización de este artículo ha presidido, al menos en parte, una concepción más moderna de las competencias regionales, en la medida en que se han intentado superar el concepto de instrucción como función del solo Estado-persona. Pero las regiones han encontrado obstáculos en el desarrollo de sus funciones en los primeros años después de su institucionalización ${ }^{49}$.

El artículo 34, en lo que afecta al derecho a la instrucción y al estudio, responde a una concepción nueva de la escuela en comparación al ordenamiento precedente. El derecho a la instrucción (o al estudio) no es solamente el derecho de ser admitido en el sistema escolar. Decir que la enseñanza elemental es obligatoria y gratuita no es, en sí mismo, un principio innovador. Respecto del pasado, sólo la extensión de la obligatoriedad escolar podría ser, parcialmente considerada, como una innovación (art. 34.2).

Lo que constituye un verdadero elemento de novedad es la obligación de la República de hacer efectivo el libre acceso a todos los órdenes y grados de la enseñanza. Se observa, por otro lado, que la Constitución vincula a un examen de los méritos - mediante concurso- la concesión de las necesarias provisiones para permitir el acceso a los «grados superiores de estudio» (art. 34.3) a aquellos que no dispongan de otros medios. Hay quien ha acusado al constituyente, aunque estuviese integrado también por partidos de izquierda, «de una concepción aristocrática de la enseñanza, que cristaliza en un criterio meritocrático de selección" 50 . Los hay que proponen la interpretación del artículo 34 de la Constitución en el sentido de reconocer, también a aquellos que superan apenas las pruebas de examen, el derecho a alcanzar los más altos grados de la enseñanza ${ }^{51}$. Es ésta una interpretación poco persuasiva, ni siquiera admi-

49 Sobre este punto, ver, por ejemplo, L. DI MARCo, «Regionali e diritto allo studio: dalla legge 16 maggio $1970 \mathrm{n}$. 281 al DPR 24 luglio 1977, n. 616, en Studi parlamentari e di politica costituzionale $63^{1}, 1984$, págs. 30 y ss.

so Ver A. MurA, ob. cit., págs. 253 y ss. Sobre el carácter no innovador de la constitución, ver también F. Staderini, ob. cit., págs. 677 y ss. De distinta opinión V. ATRIPaldi, Diritto allo studio, Napoli, 1974, págs. 43 y ss.

51 Ver V. Ataipaldi, ob. cit., pág. 267; F. Fenucci, L'assistenza scolastica nelle leggi delle regioni ad autonomia ordinaria, Napoli, 1976, pág. 39. 
tiendo que el entendimiento del artículo 34 de la Constitución se encuentra vinculado estrechamente a lo dispuesto ahora sobre la igualdad sustancial que implica la instauración de las nuevas relaciones entre la escuela y la sociedad.

Apuntemos que las disposiciones referentes a la escolaridad no han de entenderse -en conexión con el artículo 3.2 de la Constitucióncomo intervenciones de la beneficencia, sino de la seguridad social. Se trata de derechos y no de caridad.

La escolarización, como se deduce del DPR n. 3 de 1972 y del posterior DPR n. 616, se basaba en intervenciones exclusivamente económicas, pero las intervenciones regionales han ido más allá de estos límites. Actividades preescolares, senvicios psico-pedagógicos de orientación, formación del profesorado, servicio de mantenimiento y recuperación. Obviamente ello supone una interpretación extensiva de las competencias regionales, en el caso de que se mantenga que la escolarización es una actividad restringida a la intervención económica. No obstante, como ha observado OrSIBATTAGLINI, "es posible que, en el futuro, pueda llegarse a superar la distinción entre la enseñanza como función estatal y la escolarización como función regional y local, concibiéndose así la escuela como una estructura organizativa en la que confluyan instrucción y escolarización".

Indiquemos que el término "escolarización» no se utiliza en los estatutos regionales, que usan preferentemente fórmulas como "derecho a la enseñanza" y "condiciones para hacer efectivo el derecho a la enseñanza" (Est. Piamonte, art. 4.4; Est. Toscana, art. 4.1; Est. Marche, art. 5.2.).

Para concluir, digamos que las regiones de estatuto especial y, en el Trentino-Alto Adigio, las provincias de Trento y Bolzano, tienen competencias diferenciadas en el sector educativo respecto de las otras regiones. De este modo, por ejemplo, los estatutos siciliano, valdostano y el del Trentino-Alto Adigio contemplan una competencia legislativa exclusiva o primaria en las materias de instrucción elemental, enseñanza técnicoprofesional y post elemental y de capacitación profesional. La región sarda tiene una competencia integradora para todos los grados y órdenes de la enseñanza. La región siciliana tiene capacidad legislativa concurrente en el campo de la enseñanza media y universitaria. En el Trentino-Alto Adigio la potestad legislativa en el sector educativo está limitada a las dos provincias de Trento y Bolzano. 